ACCEPTED FOR PUBLICATION IN APJ, 7 APRIL 2006

Preprint typeset using $\mathrm{LT}_{\mathrm{E}} \mathrm{X}$ style emulateapj v. 6/22/04

\title{
SYNTHESIS IMAGING OF DENSE MOLECULAR GAS IN THE N113 H II REGION OF THE LARGE MAGELLANIC CLOUD
}

\author{
TONY WONG ${ }^{1}$, JOHN B. WhITEOAK, \& JÜRGEN OTT \\ CSIRO Australia Telescope National Facility, PO Box 76, Epping NSW 1710, Australia \\ YI-NAN CHIN \\ Department of Physics, Tamkang University, 25137 Tamsui, Taipei, Taiwan \\ AND \\ MARIA R. CUNNINGHAM \\ School of Physics, University of New South Wales, Sydney NSW 2052, Australia \\ Accepted for publication in ApJ, 7 April 2006
}

\begin{abstract}
We present aperture synthesis imaging of dense molecular gas in the Large Magellanic Cloud, taken with the prototype millimeter receivers of the Australia Telescope Compact Array (ATCA). Our observations of the N113 H II region reveal a condensation with a size of $\sim 6^{\prime \prime}$ (1.5 pc) FWHM, detected strongly in the 1-0 lines of $\mathrm{HCO}^{+}, \mathrm{HCN}$ and $\mathrm{HNC}$, and weakly in $\mathrm{C}_{2} \mathrm{H}$. Comparison of the ATCA observations with singledish maps from the Mopra Telescope and sensitive spectra from the Swedish-ESO Submillimetre Telescope indicates that the condensation is a massive clump of $\sim 10^{4} M_{\odot}$ within a larger $\sim 10^{5} M_{\odot}$ molecular cloud. The clump is centered adjacent to a compact, obscured H II region which is part of a linear structure of radio continuum sources extending across the molecular cloud. We suggest that the clump represents a possible site for triggered star formation. Examining the integrated line intensities as a function of interferometer baseline length, we find evidence for decreasing $\mathrm{HCO}^{+} / \mathrm{HCN}$ and $\mathrm{HCN} / \mathrm{HNC}$ ratios on longer baselines. These trends are consistent with a significant component of the $\mathrm{HCO}^{+}$emission arising in an extended clump envelope and a lower HCN/HNC abundance ratio in dense cores.
\end{abstract}

Subject headings: Magellanic Clouds_-ISM: molecules_-ISM: structure_-galaxies: ISM

\section{INTRODUCTION}

Cold molecular gas in the interstellar medium (ISM) is usually studied via rotational transitions of trace molecules that emit at millimeter and sub-millimeter wavelengths. The most commonly used tracer is $\mathrm{CO}$, which typically traces gas densities of $\sim 10^{2}-10^{3} \mathrm{~cm}^{-3}$. Higher densities are best probed using molecules with larger dipole moments such as $\mathrm{HCO}^{+}$ and HCN. Emission from these molecules comes from the densest condensations within molecular clouds, often referred to as "clumps" and "cores" (e.g., Blitz 1991). A molecular core has typical radius $R \sim 0.1 \mathrm{pc}$, mass $M \sim 1-10 M_{\odot}$, and density $n \gtrsim 10^{4} \mathrm{~cm}^{-3}$, and is thought to eventually form a single star or multiple star system. Clumps are somewhat larger structures which may evolve into star clusters. The use of high-density tracers to study the initial conditions for star formation has long been common practice for Galactic observers, and has recently gained greater prominence in studies of nearby galaxies following the demonstration of a tight correlation between HCN and far-infrared (FIR) luminosities (Gao \& Solomon 2004b), the latter tracing recent star formation.

Although they have similar dipole moments, $\mathrm{HCO}^{+}$and $\mathrm{HCN}$ may not trace dense gas equally well, as one must consider the chemical processes which lead to their formation and destruction. This becomes especially apparent when examining molecular abundances in the Magellanic Clouds, where a lower dust-to-gas ratio and locally active star formation ac-

\footnotetext{
Electronic address: Tony.Wong@csiro.au

${ }^{1}$ also at School of Physics, University of New South Wales, Sydney NSW 2052, Australia
}

tivity combine to yield a stronger photoionizing flux than in the Galaxy. As a result, molecular clouds are immersed in extensive photon-dominated regions (PDRs) where carbon is mostly ionized, as evidenced by the much higher [C II]/CO intensity ratios in the LMC (Mochizuki et al. 1994; Israel et al. 1996). While the formation of $\mathrm{HCO}^{+}$in regions shielded from far-ultraviolet (FUV) radiation is dominated by reactions between $\mathrm{H}_{3}^{+}$and $\mathrm{CO}$, it can also occur in PDRs via reactions between $\mathrm{C}^{+}$and $\mathrm{OH}, \mathrm{O}_{2}$, and $\mathrm{H}_{2} \mathrm{O}$ (Graedel et al. 1982). Thus, the spatial extent of the $\mathrm{HCO}^{+}$emission may be much larger than that of $\mathrm{HCN}$ - even comparable to that of $\mathrm{CO}$.

The HNC molecule, on the other hand, has been suggested to be a tracer of cold cores, as steady-state chemical models predict that it will be much weaker than $\mathrm{HCN}$ in regions of high temperature and density (Schilke et al. 1992). Consistent with these predictions, the $\mathrm{HCN} / \mathrm{HNC}$ abundance ratio is particularly high $(\approx 80)$ in the hot core of Orion KL (Schilke et al. 1992), whereas it is close to unity in cool dark clouds (Churchwell et al. 1984; Hirota et al. 1998). However, observations of starburst galaxies, where temperatures are expected to be high, have revealed HNC intensities that are sometimes close to those of HCN (Huettemeister et al. 1995; Aalto et al. 2002). While an extended component of cool, quiescent gas could contribute to the HNC emission, Aalto et al. (2002) speculate that PDR chemistry may play a role: reactions involving $\mathrm{HCNH}^{+}$may become important, which recombines to produce $\mathrm{HCN}$ and $\mathrm{HNC}$ with equal probability and largely independent of kinetic temperature. Thus, HNC is potentially another tracer of PDR conditions.

As one of the initial targets for the prototype millimeter re- 
ceivers on the Australia Telescope Compact Array ${ }^{2}$ (ATCA), we have observed the H II region N113 in the Large Magellanic Cloud (LMC) in the $3 \mathrm{~mm}$ transitions of $\mathrm{HCO}^{+}, \mathrm{HCN}$, $\mathrm{HNC}$, and $\mathrm{C}_{2} \mathrm{H}$. The LMC's proximity $(d=52 \mathrm{kpc}$, Panagia 1999) and low metallicity make it a very attractive target for studies of the molecular ISM, but its southern declination has heretofore made it inaccesible to millimeter interferometry. We use $\mathrm{HCN}$ as a fiducial dense gas tracer and examine the relative intensities of the other molecular lines (including $\mathrm{C}_{2} \mathrm{H}$, produced in reactions involving $\mathrm{C}^{+}$) for enhancements due to the presence of a PDR. In particular, we examine the line ratios as a function of spatial scale, which allows a comparison of compact and extended emission regions.

The molecular cloud associated with N113 (Henize 1956) is one of the richest in the LMC, with a peak $\mathrm{CO}(1-0)$ brightness temperature (measured with the $45^{\prime \prime}$ beam of the Swedish-ESO Submillimetre Telescope, SEST) of $\sim 9$ $\mathrm{K}$ (Chin et al. 1997). Also associated with N113 are three young ( $<10 \mathrm{Myr}$ ) stellar clusters, NGC 1874, NGC 1876, and NGC 1877 (Bica et al. 1992). Current star formation activity appears concentrated in three compact radio continuum sources, superposed on an extended emission component and aligned in a northwest-southeast direction with separations of $\sim 20^{\prime \prime}$ (Brooks \& Whiteoak 1997). The faintest, easternmost source contains by far the brightest $\mathrm{H}_{2} \mathrm{O}$ maser in the LMC (Whiteoak \& Gardner 1986; Lazendic et al. 2002) as well as an $\mathrm{OH}$ maser (Brooks \& Whiteoak 1997). The brightest, westernmost source also shows $\mathrm{H}_{2} \mathrm{O}$ maser emission (Lazendic et al. 2002); it is towards this source that we have targeted our ATCA observations. All four of the $3 \mathrm{~mm}$ transitions observed with ATCA have been previously detected in this region with the SEST. In this paper, we present the ATCA results and compare them with single-dish results from the Mopra and SEST telescopes.

\section{OBSERVATIONS}

\subsection{ATCA 3-mm data}

Observations with the ATCA were made in 2003 July and August in the EW214 and EW367 configurations respectively. At the time of the observations, the ATCA had three antennas of $22 \mathrm{~m}$ diameter equipped with dual polarisation 3-mm receivers covering the bands $84.9-87.3$ and $88.5-91.3 \mathrm{GHz}$. At these frequencies the primary beam has a FWHM of $36^{\prime \prime}$. Both configurations had all three antennas arranged in an eastwest line. The EW214 configuration provided baselines of $30 \mathrm{~m}, 75 \mathrm{~m}$, and $105 \mathrm{~m}$, whereas the EW367 provided baselines of $45 \mathrm{~m}, 90 \mathrm{~m}$, and $135 \mathrm{~m}$. The pointing center for the observations was $\alpha_{2000}=5^{\mathrm{h}} 13^{\mathrm{m}} 18^{\mathrm{s}}, \delta_{2000}=-69^{\circ} 22^{\prime} 20^{\prime \prime}$. The ATCA correlator can be configured to observe two frequencies simultaneously, and we cycled between 3 pairs of frequencies during each observing session in order to obtain comparable coverage of the visibility plane. The first pair of frequencies consisted of the $\mathrm{HCO}^{+}(J=1-0)$ and $\mathrm{HNC}(J=1-0)$ lines at 89.1885 and $90.6635 \mathrm{GHz}$ respectively, redshifted to the appropriate observatory frequency for $v_{\mathrm{LSR}}=235 \mathrm{~km} \mathrm{~s}^{-1}$, the second pair consisted of the $\mathrm{HCN}(J=1-0)$ line at $88.6318 \mathrm{GHz}$ and $\mathrm{HNC}$, and the third pair consisted of the $\mathrm{C}_{2} \mathrm{H}(N=1-0$, $J=3 / 2-1 / 2, F=2-1$ ) line at $87.3169 \mathrm{GHz}$ and a $128 \mathrm{MHz}$ wide continuum window at $86.243 \mathrm{GHz}$. Each line was observed with a channel spacing of $0.5 \mathrm{MHz}$ across a $64 \mathrm{MHz}$ bandwidth, except for $\mathrm{C}_{2} \mathrm{H}$, which was observed with a channel

\footnotetext{
2 The Australia Telescope is funded by the Commonwealth of Australia for operation as a National Facility managed by CSIRO.
}

TABLE 1

Properties OF THE ATCA IMAGES

\begin{tabular}{lccccccc} 
Line & $\begin{array}{c}\text { Beam size Beam PA } \\
\left({ }^{\prime \prime} \times{ }^{\prime \prime}\right)\end{array}$ & $\begin{array}{c}\left.\sigma_{\mathrm{ch}^{\circ}}{ }^{\circ}\right) \\
\left(\mathrm{mJy} \mathrm{bm}^{-1}\right)\end{array}$ & $\begin{array}{c}\sigma_{\mathrm{ch}} \\
(\mathrm{K})\end{array}$ & $\begin{array}{c}T_{\text {peak }} \\
(\mathrm{K})\end{array}$ & $\begin{array}{c}\text { Vel. range } \\
\left(\mathrm{km} \mathrm{s}^{-1}\right)\end{array}$ & $\begin{array}{c}\text { Flux } \\
\left(\mathrm{Jy} \mathrm{km} \mathrm{s}^{-1}\right)\end{array}$ \\
\hline $\mathrm{HCO}^{+}$ & $5.1 \times 3.2$ & -9 & 41 & 0.38 & 4.2 & {$[229,241]$} & $5.2-8.8$ \\
$\mathrm{HCN}$ & $5.0 \times 3.4$ & 0 & 42 & 0.39 & 2.8 & {$[223,243]$} & $4.1-6.9$ \\
$\mathrm{HNC}$ & $4.9 \times 3.3$ & -5 & 23 & 0.21 & 1.4 & {$[229,241]$} & $1.0-2.1$ \\
$\mathrm{C}_{2} \mathrm{H}$ & $4.7 \times 3.6$ & -5 & 49 & 0.46 & 1.1 & {$[233,239]$} & $0.5-1.5$ \\
Cont. & $4.7 \times 3.7$ & -4 & 3 & 0.03 & 0.3 & - & $0.04 \mathrm{Jy}$ \\
\hline
\end{tabular}

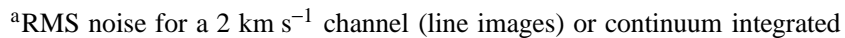
across $84 \mathrm{MHz}$.

${ }^{b}$ Flux measurements are made in boxes of $10^{\prime \prime} \times 10^{\prime \prime}$ and $20^{\prime \prime} \times 20^{\prime \prime}$ centered on $\alpha_{2000}=5: 13: 17.2, \delta_{2000}=-69: 22: 23$.

spacing of $0.0625 \mathrm{MHz}$ across $16 \mathrm{MHz}$. Figure 1 a) shows the coverage of the visibility plane obtained for the $\mathrm{HCO}^{+}$ line.

The data were calibrated and imaged with the MIRIAD package (Sault et al. 1995), which at ATNF has been updated with new procedures to handle ATCA 3-mm data. Flux calibration was performed using Uranus, while the quasars B0537-441 and B1253-055 (3c279) were used for gain and bandpass calibration respectively. However, for the last frequency pair $\left(\mathrm{C}_{2} \mathrm{H}\right.$ and continuum), the $\mathrm{SiO}$ maser $\mathrm{R}$ Dor was used for gain calibration instead; in doing so the maser flux was assumed to be constant over the course of an $\sim 8$ hour observation. An instrumental phase error causing phase discontinuities when changing sources had been identified with ATCA 3-mm data, ${ }^{3}$ and we compensated for this using an antenna position correction which was determined by observations of multiple quasars within a few days of the observations. We produced channel maps spaced in velocity by 2 $\mathrm{km} \mathrm{s}^{-1}$, which is close to the effective resolution provided by the correlator in the $\mathrm{HCN}, \mathrm{HNC}$, and $\mathrm{HCO}^{+}$lines. The phase center was shifted to $\alpha_{2000}=5^{\mathrm{h}} 13^{\mathrm{m}} 17^{\mathrm{s}} 2, \delta_{2000}=-69^{\circ} 22^{\prime} 23^{\prime \prime}$ before imaging, a shift of $5^{\prime \prime}$ (this shift has negligible impact on the primary beam response at the locations of detected emission). The noise level and synthesized beamwidth for each map are given in Table 1 After imaging the maps were CLEANed to a $2 \sigma$ level.

\subsection{ATCA 1.3-cm data}

Additional observations were obtained with ATCA in 2005 March using the recently installed 12-mm receivers. N113 was observed in the H214 configuration (baseline lengths $80 \mathrm{~m}-250 \mathrm{~m}$ ) over two days with a total integration time of $160 \mathrm{~min}$. The correlator was configured for 512 channels across $8 \mathrm{MHz}$ (velocity resolution $0.24 \mathrm{~km} \mathrm{~s}^{-1}$ ) at $23.673 \mathrm{GHz}$ and 32 channels across $128 \mathrm{MHz}$ at $23.9 \mathrm{GHz}$. The narrowband observations were designed to detect the $\mathrm{NH}_{3}(1,1)$ transition but failed to do so at an RMS noise level of 20 $\mathrm{mJy} \mathrm{bm}{ }^{-1}$. The wideband observations, used to image the continuum, achieved a noise level of $\sim 0.4 \mathrm{mJy} \mathrm{bm}^{-1}$. Flux, bandpass, and phase calibration were performed using the quasars B1934-638, B1921-293, and B0454-810 respectively, following standard reduction procedures in MIRIAD. After Fourier inversion the $1.3-\mathrm{cm}$ continuum image was CLEANed

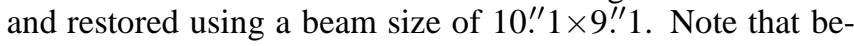
cause of the limited coverage of the Fourier plane achieved with the H214 configuration, structure on scales much larger

\footnotetext{
3 This is believed to be an instrumental error which corrupts the antenna baseline solution.
} 
$u(k \lambda) \quad \Delta \alpha(\operatorname{arcsec})$
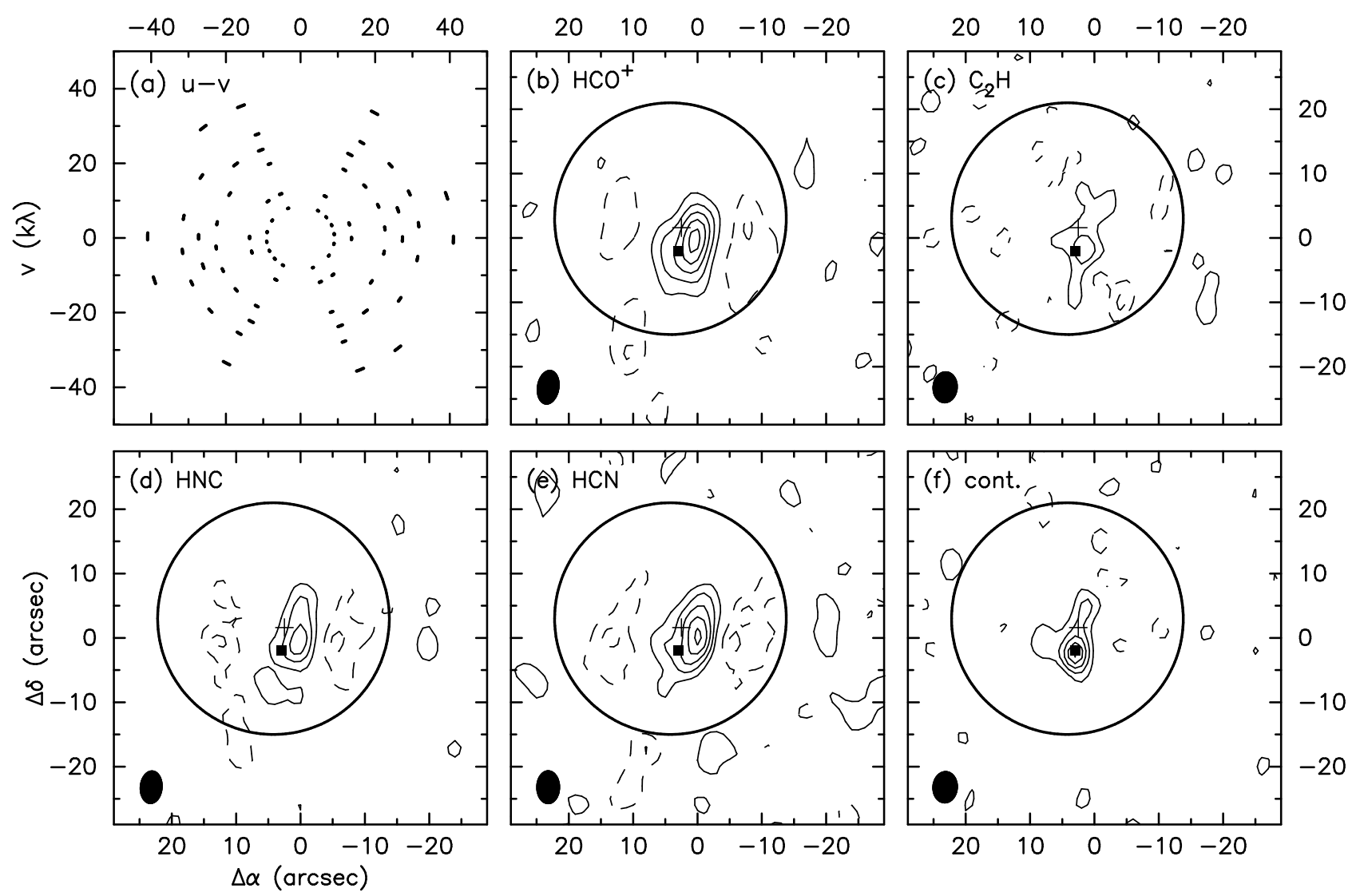

FIG. 1.- Summary of ATCA observations towards N113. (a) Coverage of the visibility plane for $\mathrm{HCO}^{+}$obtained from combining both runs. Other panels show integrated intensity maps for (b) $\mathrm{HCO}^{+}$; (c) $\mathrm{C}_{2} \mathrm{H}$; (d) $\mathrm{HNC}$; (e) $\mathrm{HCN}$; (f) continuum. Offsets are relative to $\alpha_{2000}=5^{\mathrm{h}} 13^{\mathrm{m}} 17^{\mathrm{s}} 2, \delta_{2000}=-69^{\circ} 22^{\prime} 23^{\prime \prime}$. The synthesized beam FWHM is shown in the lower left corner and the large circle represents the FWHM field of view. The solid square and cross represent the positions of the $K$-band source and the weaker $\mathrm{H}_{2} \mathrm{O}$ maser respectively. The contour levels are spaced by $6 \mathrm{~K} \mathrm{~km} \mathrm{~s}^{-1}$ for $\mathrm{HCO}^{+}$and $\mathrm{HCN}^{3} 3 \mathrm{~K} \mathrm{~km} \mathrm{~s}^{-1}$ for $\mathrm{HNC}$ and $\mathrm{C}_{2} \mathrm{H}$, and $0.06 \mathrm{~K}$ for the continuum.

than $30^{\prime \prime}$ will be poorly imaged. Thus, the resulting image is dominated by compact ionized regions, and the extended continuum emission from the large-scale $\mathrm{H}$ II region was not detected.

\subsection{SEST data}

Although most of our comparison with SEST data makes use of values previously presented by Chin et al. (1997), we also made use of some data which had not yet been published. These consisted of small grid maps made in the $\mathrm{HCN}(J=1 \rightarrow 0)$ and $\mathrm{HCO}^{+}(J=1 \rightarrow 0)$ lines in two observing runs with the SEST in 1997 January and July. The maps cover a region of roughly $1.5 \times 1.5$ centered on the position $\alpha_{2000}=5^{\mathrm{h}} 13^{\mathrm{m}} 18.2, \delta_{2000}=-69^{\circ} 22^{\prime} 35^{\prime \prime}$. The grid spacing was $20^{\prime \prime}$ (for comparison the SEST beam has a FWHM of $58^{\prime \prime}$ ), but the grids were not completely sampled in the outer parts. A $3 \mathrm{~mm}$ SIS receiver was used in single-sideband mode with a 2000 channel acousto-optical spectrometer having a $43 \mathrm{kHz}$ channel separation.

A dual beam switching mode, where the source is placed alternately in the main and reference beams, was used to remove the sky background, and the spectra were placed on a $T_{\mathrm{MB}}$ scale using a main beam efficiency of 0.76 . Typical single-sideband (SSB) system temperatures were $\sim 200 \mathrm{~K}$. The CLASS package was used to calibrate and baseline the spectra. For purposes of this paper we have produced high signal-to-noise spectra for both lines by averaging the 9 spectra within $-20^{\prime \prime}<\Delta \alpha<20^{\prime \prime}$ and $-20^{\prime \prime}<\Delta \delta<20^{\prime \prime}$ relative to the center position. The corresponding maps show only a moderately resolved central source and are not reproduced here. Further details of the SEST observations will be presented in a forthcoming paper (M. Wang et al., in preparation).

\subsection{Mopra data}

A $4^{\prime} \times 4^{\prime}$ region centered on the position $\alpha_{2000}=5^{\mathrm{h}} 13^{\mathrm{m}} 18^{\mathrm{s}}$, $\delta_{2000}=-69^{\circ} 22^{\prime} 25^{\prime \prime}$ was mapped in the $J=1 \rightarrow 0$ transitions of $\mathrm{HCO}^{+}, \mathrm{HCN}$, and $\mathrm{CO}$ with the 22-m ATNF Mopra telescope in three runs from 2004 June to September. The newly implemented On-the-Fly (OTF) mode was used, in which the telescope takes data continuously while moving across the sky. Spectra were spaced by $7^{\prime \prime}$ so that the $36^{\prime \prime}$ Mopra beam $\left(33^{\prime \prime}\right.$ at $\mathrm{CO})$ would be well oversampled in the scanning direction; the row spacing was $10^{\prime \prime}$, also oversampling the beam. Each row was preceded by an off-source integration at a location about $10^{\prime}$ from the map centre. Typical SSB $T_{\text {sys }}^{*}$ values of $200 \mathrm{~K}$ for $\mathrm{HCO}^{+}$and $\mathrm{HCN}$ and $600 \mathrm{~K}$ for $\mathrm{CO}$ were obtained in clear conditions. The pointing was checked on the $\mathrm{SiO}$ maser $\mathrm{R}$ Dor prior to each map; typical corrections were less than $5^{\prime \prime}$. 

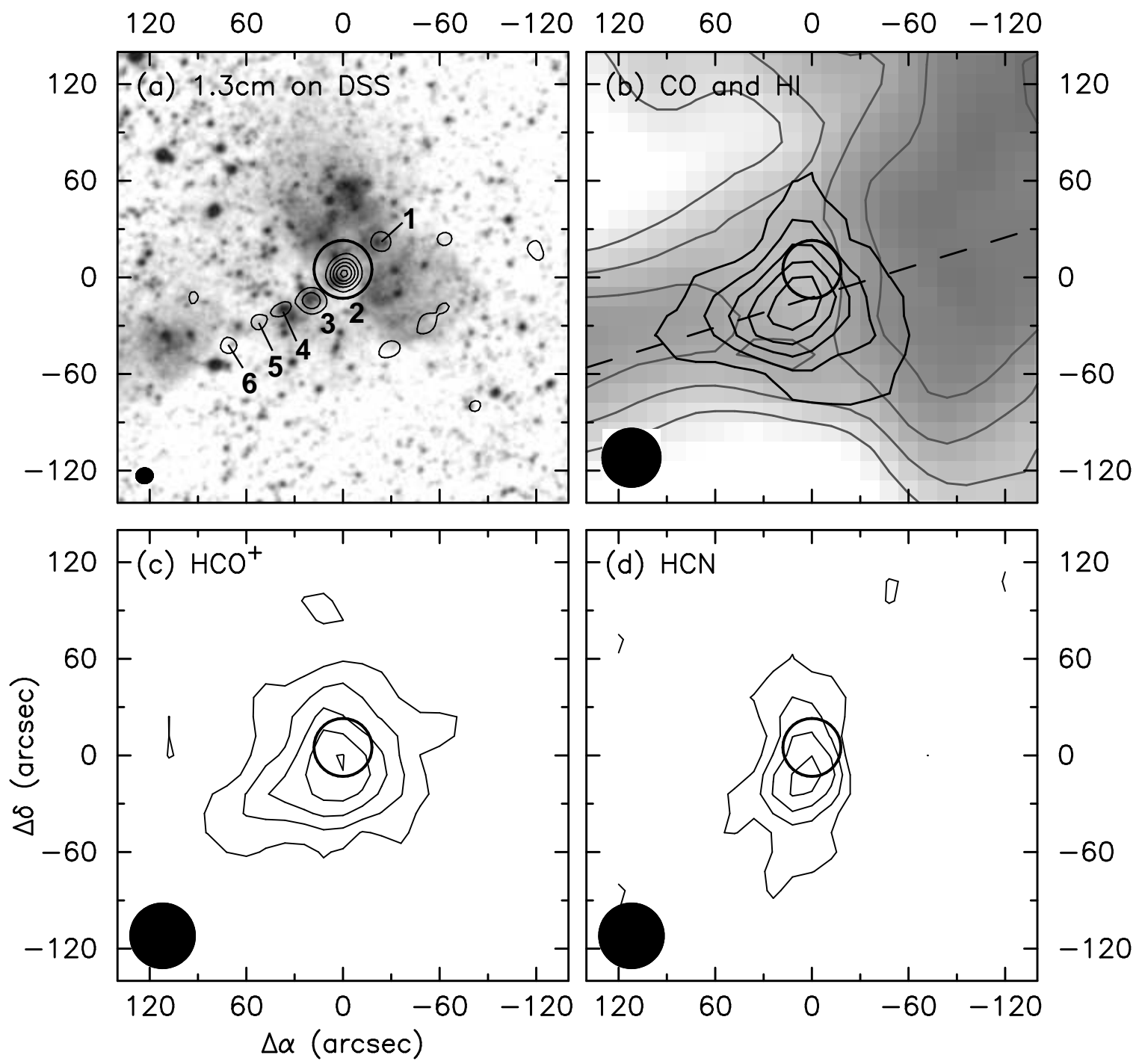

FIG. 2.- (a) ATCA $1.3 \mathrm{~cm}$ map (contours) overlaid on an $R$-band image from the Digitized Sky Survey. Contour levels correspond to $2 n^{2} \mathrm{mJy}$ bm ${ }^{-1}(n=1,2, \ldots)$. (b) Mopra CO (1-0) map (black contours) overlaid on an H I peak intensity image (grayscale and gray contours). Contour levels are $10 \mathrm{~K} \mathrm{~km} \mathrm{~s}^{-1}$ for the CO map and 40,50, .., $80 \mathrm{~K}$ for the $\mathrm{H} \mathrm{I}$ map. The dashed line represents the position-velocity slice shown in Fig. 3 (c) Mopra $\mathrm{HCO}^{+}(1-0)$ map. Contour levels are spaced by $0.6 \mathrm{~K} \mathrm{~km} \mathrm{~s}^{-1}$. (d) Mopra HCN (1-0) map. Contour levels are spaced by $0.5 \mathrm{~K} \mathrm{~km} \mathrm{~s}^{-1}$. In each case the solid open circle represents the ATCA pointing position. Offsets are relative to $\alpha_{2000}=5^{\mathrm{h}} 13^{\mathrm{m}} 18^{\mathrm{s}}, \delta_{2000}=-69^{\circ} 22^{\prime} 25^{\prime \prime}$.

The digital correlator was configured to output 1024 channels across $64 \mathrm{MHz}$ in each of two orthogonal polarisations, except for the June observations where only one polarisation was recorded. Initial spectral processing (baselining and calibration onto a $T_{A}^{*}$ scale) was performed using the LiveData task in AIPS++, and the spectra were gridded into datacubes using the AIPS++ Gridzilla task. During the gridding, a Gaussian smoothing kernel with a FWHM half that of the beamsize was convolved with the data, so the effective resolutions of the final cubes were $40^{\prime \prime}$ for $\mathrm{HCO}^{+}$and $\mathrm{HCN}$ and $36^{\prime \prime}$ for $\mathrm{CO}$. The cubes were then rescaled onto a $T_{\mathrm{MB}}$ scale using an "extended beam" efficiency of $\eta_{\mathrm{xb}}=0.55$ for $\mathrm{CO}$ and 0.65 for $\mathrm{HCO}^{+}$and HCN (Ladd et al. 2005). This takes into account that sources larger than about $80^{\prime \prime}$ in diameter will couple to both the main beam and the inner error beam of the telescope.

Comparison of Mopra and SEST spectra taken at the central Mopra position, with the Mopra maps convolved to the SEST resolution, indicate that the Mopra line intensities are lower by $\sim 30 \%$. This is not surprising given that the extended beam efficiency is higher than the main beam efficiency $\eta_{\mathrm{mb}}$ by $\sim 30 \%$ (Ladd et al. 2005). While use of $\eta_{\mathrm{mb}}$ rather than $\eta_{\mathrm{xb}}$ would place the Mopra data on a more accurate temperature scale for compact sources, it would lead to an overestimate of the total map flux when assuming a Gaussian beam size equal to the main beam. Thus we continue to use $\eta_{\mathrm{xb}}$ to calibrate the spectra, noting however that the quoted beam size is somewhat less than the effective beam size due to the presence of a significant inner error beam.

\section{ANALYSIS \& RESULTS}

\subsection{The N113 Molecular Cloud}

We begin by presenting maps and derived properties for the molecular cloud as a whole, based on the Mopra data. We discuss the high-resolution ATCA 3-mm data in $\$ 3.2$

\subsubsection{ATCA 1.3-cm and Mopra maps}

Figure 2]a) shows the ATCA 1.3-cm continuum image overlaid on a red Digitized Sky Survey image showing 
TABLE 2

PARAMETERS OF GAUSSIAN FITS TO MOPRA IMAGES

\begin{tabular}{lccccc} 
Line & $\begin{array}{c}\text { Center RA } \\
(\text { J2000) }\end{array}$ & $\begin{array}{c}\text { Center DEC } \\
(\text { J2000) }\end{array}$ & $\begin{array}{c}\text { Major axis } \\
\left({ }^{\prime}{ }^{\text {a }}\right)\end{array}$ & $\begin{array}{c}\text { Minor axis } \\
\left({ }^{\prime}\right)\end{array}$ & $\begin{array}{c}\text { Position angle } \\
\left({ }^{\circ}\right)\end{array}$ \\
\hline $\mathrm{CO}$ & 51319.9 & -692243 & $81 \pm 2$ & $67 \pm 2$ & -80 \\
$\mathrm{HCO}^{+}$ & 51319.4 & -692232 & $89 \pm 3$ & $67 \pm 2$ & -59 \\
$\mathrm{HCN}^{5}$ & 51319.3 & -692233 & $84 \pm 4$ & $25 \pm 2$ & -4
\end{tabular}

${ }^{a}$ Deconvolved using a Gaussian beam of $36^{\prime \prime} \mathrm{FWHM}$ for $\mathrm{CO}$ and $40^{\prime \prime}$ for $\mathrm{HCO}^{+}$and $\mathrm{HCN}$.

much of the diffuse $\mathrm{H} \alpha$ emission from the $\mathrm{H}$ II region. In addition to the string of three bright sources reported by Brooks \& Whiteoak (1997), labelled 2, 3, and 4, we have detected three additional continuum sources along the same formation, one west of the main source (Source 1) and two to the southeast (Sources 5 and 6). The fluxes of the six continuum sources, from west to east, are estimated based on Gaussian fits to be $6.2,66,18,4.1,3.9$, and $4.4 \mathrm{mJy}$. Measuring the flux by direct summation of image pixels yields fluxes consistent with these values within $1 \mathrm{mJy}$. Apparent sources which lie outside this linear structure (e.g., southwest of the image center) are likely to be sidelobes that were incompletely removed.

Figures [2] b)-(d) show the Mopra $\mathrm{CO}, \mathrm{HCO}^{+}$, and $\mathrm{HCN}$ maps integrated from a velocity of 231 to $240 \mathrm{~km} \mathrm{~s}^{-1}$. Remarkably, at the resolution of Mopra the molecular cloud appears to be a centrally condensed structure, in contrast to the filamentary structure which is characteristic of the $\mathrm{H}$ I clouds in the LMC (Kim et al. 2003). The CO map is shown overlaid on $\mathrm{H}$ I emission contours from the combined Parkes/ATCA survey (Kim et al. 2003). The $\mathrm{HCO}^{+}$emission imaged with Mopra shows a similar extent to the $\mathrm{CO}$, however the peak intensity is shifted $\sim 10^{\prime \prime}$ to the north. The HCN emission appears to be narrower in the east-west direction than the other tracers, as confirmed by fitting of two-dimensional Gaussians to the images (Table 2).

\subsubsection{Mass and Dynamical Properties}

We can estimate the mass of the molecular cloud from the $\mathrm{CO}$ data in two ways. Adopting a conversion from $\mathrm{K}$ to $\mathrm{Jy}$ of $1 \mathrm{~K}=9.6 \times 10^{-3} \mathrm{Jy} \operatorname{arcsec}^{-2}$, the total $\mathrm{CO}(1-0)$ line flux detected by the Mopra observations is $2.9 \times 10^{3} \mathrm{Jy}$ $\mathrm{km} \mathrm{s}^{-1}$ over a $4^{\prime} \times 4^{\prime}$ region. Using a standard (Galactic) $\mathrm{CO}$ to $\mathrm{H}_{2}$ conversion factor of $N_{\mathrm{H}_{2}} / I_{\mathrm{CO}}=2.0 \times 10^{20} \mathrm{~cm}^{-2}$ $\left(\mathrm{K} \mathrm{km} \mathrm{s}^{-1}\right)^{-1}$ (Strong \& Mattox 1996; Dame et al. 2001), this translates into a total gas mass (including $\mathrm{He}$ ) of $8.2 \times 10^{4}$ $M_{\odot}$. We can compare this with a virial mass estimate, following Heikkilä et al. (1999), of $M_{\text {vir }}=151 R_{\mathrm{CO}}(\Delta v)^{2}$, where $R_{\mathrm{CO}}$ is the deconvolved FWHM size of the cloud in pc. For N113, a Gaussian fit to the integrated CO intensity gives $R=74^{\prime \prime}(19 \mathrm{pc})$ after deconvolution, which for $\Delta v=5.2$ $\mathrm{km} \mathrm{s}^{-1}$ (the average FWHM linewidth over the inner $1^{\prime} \times 1^{\prime}$ ) yields $M_{\text {vir }}=7.8 \times 10^{4} M_{\odot}$. Although only a rough estimate, this suggests, as noted previously by Chin et al. (1997), that a standard conversion factor yields mass estimates for LMC molecular clouds that are consistent with virial mass estimates when observed at resolutions of $\sim 10 \mathrm{pc}$. A similar result was obtained for SMC clouds by Rubio et al. (1993).

Using the mass estimate above and a radius of $15 \mathrm{pc}$ (estimated as $80 \%$ of the FWHM, following Heikkilä et al. 1999) gives an overall density for the cloud of $\left\langle n_{\mathrm{H}}\right\rangle \sim 200 \mathrm{~cm}^{-3}$. This is significantly less than the critical density for excitation of $\mathrm{HCO}^{+}$and $\mathrm{HCN}\left(\sim 10^{5} \mathrm{~cm}^{-3}\right)$, indicating that the material

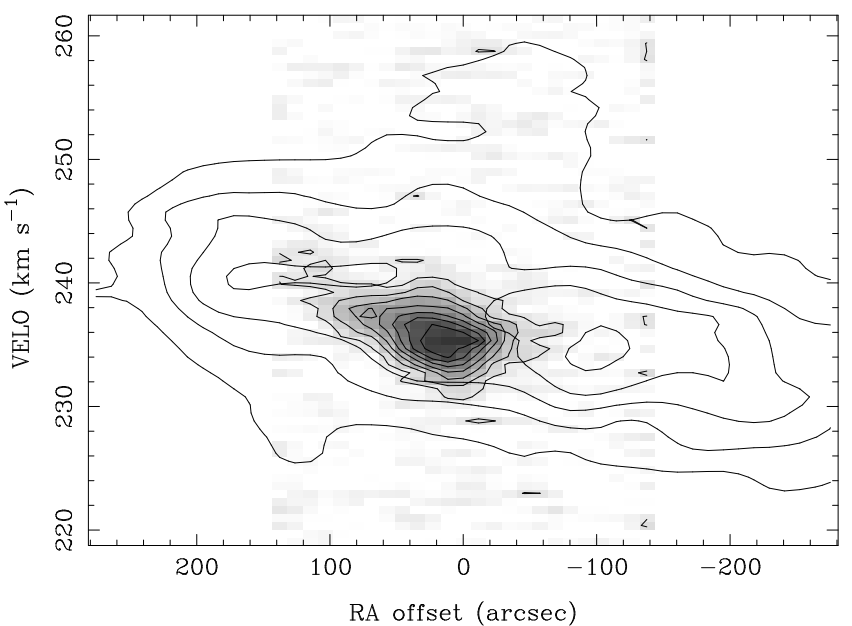

FIG. 3.- Position-velocity slice through the Mopra CO data cube (grayscale with light contours) with H I contours overlaid. The H I data have a resolution of $60^{\prime \prime}$ FWHM. The slice is centered on $\alpha_{2000}=5: 13: 18$, $\delta_{2000}=-69: 22: 43$ and has a position angle of $107^{\circ}$. Contour levels are spaced by $0.5 \mathrm{~K}$ for $\mathrm{CO}$ and $15 \mathrm{~K}$ for $\mathrm{H} \mathrm{I}$.

in the cloud is strongly clumped. Of course, our ATCA observations (described below) provide direct evidence of this clumping.

Inspection of the $\mathrm{CO}$ datacube reveals a velocity gradient of $\sim 5 \mathrm{~km} \mathrm{~s}^{-1}$ from the center to the southeast extension. The presence of such a gradient might be interpreted in terms of rotation (e.g., Rosolowsky et al. 2003). However, the CO position-velocity diagram (Figure 3) is not strongly indicative of rotation, but rather suggests a high velocity dispersion core that blends into a low velocity dispersion wing with a slightly different velocity centroid. We note that the wing emission could be composed of unresolved, clumpy structure. An overlapping slice through the H I datacube of Kim et al. (2003), smoothed to $1^{\prime}$ resolution, shows a similar velocity gradient across a filament-like structure $10^{\prime}(150 \mathrm{pc})$ in length. We conclude that the $\mathrm{CO}$ velocity gradient is not due to the rotation of a kinematically decoupled structure, but instead reflects the bulk motion of the gas from which the molecular cloud formed.

The CO cloud appears to sit within a local minimum of $\mathrm{H} \mathrm{I}$ peak brightness temperature [Fig. 20 b)]. This does not necessarily require a a large-scale conversion of the $\mathrm{H} \mathrm{I}$ into $\mathrm{H}_{2}$, as the $\mathrm{H} \mathrm{I}$ at the location of the cloud still has a high integrated intensity. Rather, the lower peak $T_{b}$ is mainly the result of a flat-topped line profile, which may indicate absorption by colder, optically thick H I.

\subsection{The Dense Embedded Clump \\ 3.2.1. ATCA 3-mm maps}

Figures 1b)-(f) show the integrated intensity maps for the four lines observed with ATCA as well as the continuum at $86 \mathrm{GHz}$. Both the line and continuum emission appear to be concentrated in a single emission peak, or "clump." These maps were produced by directly summing the velocity channels over the ranges given in Table 1 Due to the presence of large negative sidelobes in the maps, likely resulting from gain calibration errors and imperfect deconvolution, the integrated fluxes in Table 1 are given for both the inner $10^{\prime \prime} \times$ $10^{\prime \prime}$ and $20^{\prime \prime} \times 20^{\prime \prime}$ (larger values correspond to the smaller box), and should be considered indicative only. Also marked 
TABLE 3

COMPARISON OF MOPRA AND ATCA FLUXES

\begin{tabular}{|c|c|c|c|c|c|c|}
\hline Line & Mopra (tot) & Mopra (pb) & ATCA (im) & ATCA (uv) & $a_{\text {maj }}$ (uv) & $a_{\min }(\mathrm{uv})$ \\
\hline $\mathrm{CO}$ & 2900 & 320 & - & - & - & - \\
\hline $\mathrm{HCO}^{+}$ & 150 & 17 & $8.8 \pm 0.2$ & $14.2 \pm 0.9$ & $7 !^{\prime \prime} 7 \pm 0 .{ }^{\prime \prime} 8$ & $5 !^{\prime \prime} 4 \pm 0 .{ }^{\prime \prime} 8$ \\
\hline $\mathrm{HCN}$ & 73 & 10 & $6.9 \pm 0.2$ & $10.5 \pm 0.9$ & $6 .{ }^{\prime \prime} 3 \pm 2 !^{\prime \prime} 2$ & $4 !^{\prime \prime} 8 \pm 2 .{ }^{\prime \prime} 2$ \\
\hline $\mathrm{HNC}$ & - & - & $2.1 \pm 0.1$ & $3.9 \pm 0.5$ & $6 .^{\prime \prime} 8 \pm 3$ !. $0^{\prime}$ & 5 !' $^{\prime} \pm 2$. ! 4 \\
\hline $\mathrm{C}_{2} \mathrm{H}$ & - & - & $1.5 \pm 0.2$ & $3.9 \pm 1.1$ & 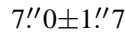 & - \\
\hline Cont. & - & - & $42 \mathrm{mJy}$ & $47 \pm 7 \mathrm{mJy}$ & $2 !^{\prime \prime} 6 \pm 0 .{ }^{\prime \prime} 6$ & - \\
\hline
\end{tabular}

NotE. - All fluxes except continuum are in Jy $\mathrm{km} \mathrm{s}^{-1}$. Mopra fluxes are derived from the total image (2nd col.) and restricted to the ATCA field of view (3rd col.). ATCA fluxes are derived from the central $10^{\prime \prime} \times 10^{\prime \prime}$ of the image and from a Gaussian fit to the visibility data. The Gaussian has been restricted to be circular for $\mathrm{C}_{2} \mathrm{H}$ and the continuum.

on each panel of Figure 1 are the positions of the obscured 2MASS point source 05131777-6922250 and the $\mathrm{H}_{2} \mathrm{O}$ maser detected by Lazendic et al. (2002). Note that the infrared source coincides with the continuum source but is offset from the peak of the molecular line emission.

\subsubsection{Flux Recovered by ATCA}

Table 3 lists the total fluxes detected in the Mopra and ATCA maps, as well as the flux detected by Mopra with the ATCA primary beam response applied (i.e., the Mopra flux restricted to the ATCA field of view). We caution that the Mopra flux within a single primary beam may be overestimated if emission outside the beam is smoothed into it; it may also be underestimated because of the use of $\eta_{\mathrm{xb}}$ to calibrate the data. In any case, we find that the ATCA flux within the central $10^{\prime \prime}$ $\times 10^{\prime \prime}$ of the integrated $\mathrm{HCO}^{+}$and HCN maps is $50-70 \%$ of the Mopra flux within the ATCA primary beam. For an independent comparison, we can also extrapolate the ATCA flux to short ("single-dish") baselines by fitting a two-dimensional Gaussian source in the visibility domain. As shown in the last three columns of Table 3, which give the fitted flux and FWHM major and minor axes, this yields a total flux that is consistent with the single-dish flux within the ATCA primary beam (assuming $\sim 20 \%$ calibration uncertainties for both telescopes). The difference compared to measuring the flux in the image plane is likely due to a more inaccurate extrapolation to short baselines provided by CLEAN, coupled with uncorrected phase errors that lead to negative regions in the deconvolved images.

The analysis above suggests that the more extended component of $\mathrm{HCO}^{+}$or $\mathrm{HCN}$ emission which does not appear in the ATCA images can be inferred by inward extrapolation of the visibility amplitude. Assuming all of the flux is recovered by the visibility plane fitting, we derive an approximate beam filling fraction (within the $36^{\prime \prime}$ ATCA beam) of $3 \%$ for the $\mathrm{HCO}^{+}$and $\mathrm{HCN}$ emission. Of course, this applies only to the region observed with the ATCA, which contains just $\sim 12 \%$ of the total flux detected by Mopra. Clearly it would be of great interest to cover the entire molecular cloud with an ATCA mosaic, to determine what fraction of the total line emission the interferometer would be sensitive to.

\subsubsection{Fitting of ATCA and SEST spectra}

While the Mopra OTF maps of $\mathrm{HCN}$ and $\mathrm{HCO}^{+}$cover a larger area, their sensitivity at any given position is a factor of 3-4 worse than the pointed SEST spectra, so we have opted to derive line widths and hyperfine ratios from the SEST data for comparison with the ATCA data.
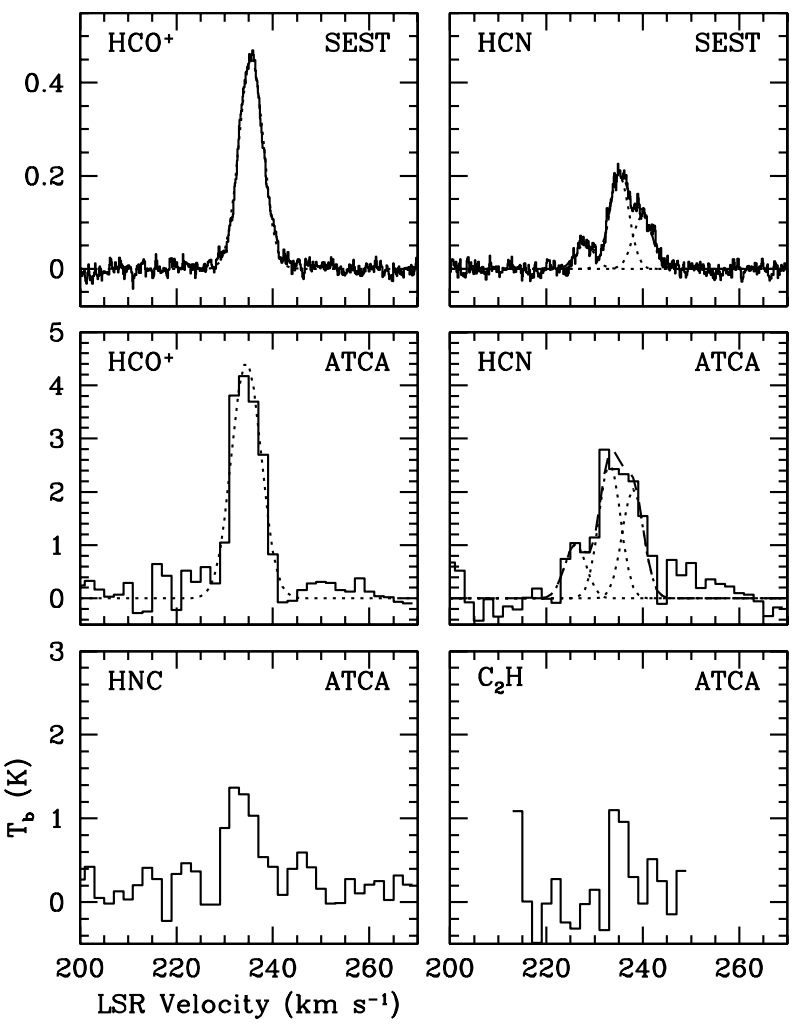

FIG. 4.- Comparison of ATCA and SEST spectra. SEST spectra, in $T_{\mathrm{mb}}$ units (top two panels), have been averaged over a $40^{\prime \prime} \times 40^{\prime \prime}$ region centred on $\alpha_{2000}=5: 13: 18, \delta_{2000}=-69: 22: 35$. Spectra through the ATCA datacubes, smoothed to $2 \mathrm{~km} \mathrm{~s}^{-1}$ and converted to brightness temperature units using the synthesized beam size, have been taken at the nominal peak position of $\alpha_{2000}=5: 13: 17.2, \delta_{2000}=-69: 22: 23$. Gaussian fits to the $\mathrm{HCO}^{+}$and $\mathrm{HCN}$ lines (the latter taking into account the spacing of the three hyperfine components) are shown as dotted lines.

The averaged SEST spectra are shown in Figure 4 along with ATCA spectra taken through the central 1" pixel of each datacube. The $\mathrm{HCO}^{+}$and $\mathrm{HCN}$ brightness temperatures $T_{b}$ measured in the ATCA maps are a factor of $\sim 10$ higher than the SEST measurements, which is not surprising given the presence of clumping and the factor of $\sim 100$ difference in beam area. We have also made single-component Gaussian fits to the $\mathrm{HCO}^{+}$spectra and three-component fits to the HCN spectra; the latter assume that the two satellite hyperfine transitions $(F=0-1$ and $F=1-1)$ are separated by -7.07 and $4.85 \mathrm{~km} \mathrm{~s}^{-1}$ from the main component and have the 


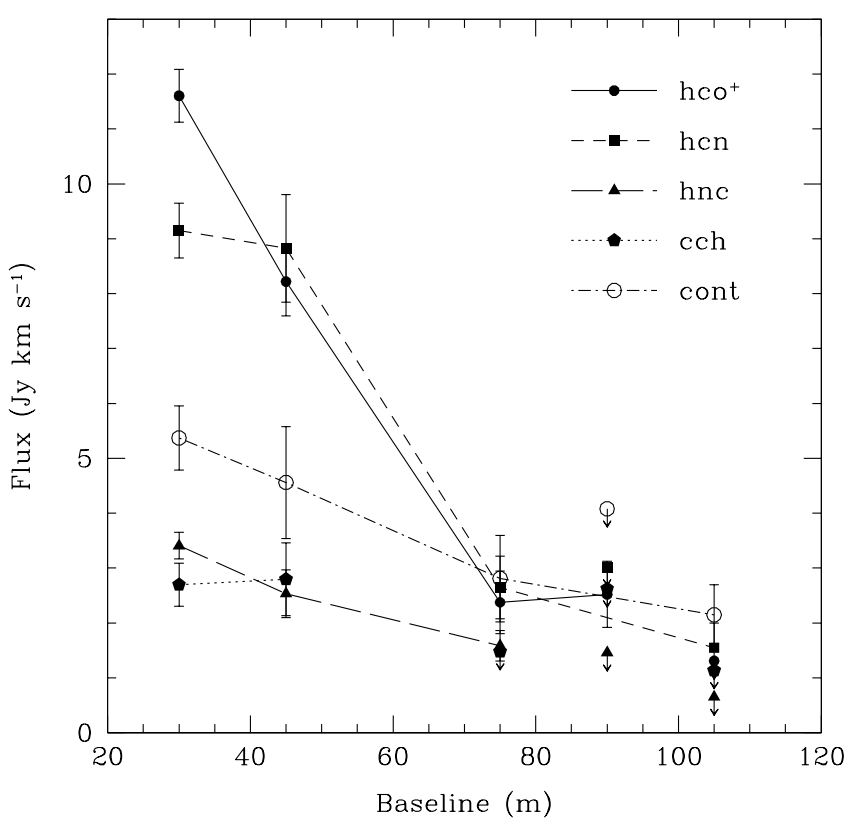

FIG. 5.- Integrated line flux for $\mathrm{HCO}^{+}, \mathrm{HCN}, \mathrm{HNC}$, and $\mathrm{C}_{2} \mathrm{H}$ as a function of projected ATCA baseline. Visibilities have been averaged within bins of $15 \mathrm{~m}$ width. For the continuum, fluxes are in units of $10 \mathrm{mJy}$. Plot symbols with arrows indicate $3 \sigma$ upper limits and are not connected with lines.

same dispersion as the main component. We find for $\mathrm{HCO}^{+}$ a FWHM linewidth (corrected for instrumental broadening) of $5.8 \mathrm{~km} \mathrm{~s}^{-1}$ for SEST and $5.5 \mathrm{~km} \mathrm{~s}^{-1}$ for ATCA. For HCN the linewidths are $4.6 \mathrm{~km} \mathrm{~s}^{-1}$ and $5.2 \mathrm{~km} \mathrm{~s}^{-1}$ for SEST and ATCA respectively, though these are much more uncertain, being based on a multi-component fit. We also find a velocity offset of $-2.0 \mathrm{~km} \mathrm{~s}^{-1}$ in the ATCA HCN spectrum relative to SEST, which we attribute to limited spectral resolution and signal-to-noise in the ATCA data.

The ratios between the hyperfine components of $\mathrm{HCN}$ can be used as a diagnostic of the optical depth, if LTE excitation is assumed (e.g., Fig. 2 in Hariu 1989). In the optically thin case, the ratio of the $F=0-1 / F=2-1$ transitions, referred to as $R_{02}$, should be 0.2 , whereas the ratio of the $F=1-1 / F=2-1$ transitions, referred to as $R_{12}$, should be 0.6 . Fitting the SEST spectrum yields $R_{02}=0.26 \pm .01$ and $R_{12}=0.57 \pm .03$, where the errors are only the formal errors on the fit and thus a lower estimate. These appear consistent with optically thin emission. On the other hand, for the ATCA spectrum we find $R_{02}=0.40 \pm .12$ and $R_{12}=0.79 \pm .19$, indicating higher opacities for the dense clump at the $1 \sigma-2 \sigma$ significance level (note however that both ratios are greater than their LTE values). While still requiring confirmation with higher spectral resolution data and/or observations of rare isotopomers, this suggests the presence of an optically thinner HCN component which dominates the total flux. We suggest that, rather than constituting a truly diffuse interclump medium, this component is an enshrouding "clump envelope" that accounts for the difference between the fluxes measured in the ATCA images and those inferred by visibility fitting (Table 3 .

\subsubsection{Clump size and line width}

The visibility fits given in Table 3 indicate that the $\mathrm{HCO}^{+}$ emission detected by the ATCA comes from a region $8^{\prime \prime} \times$ $5^{\prime \prime}(2.0 \times 1.3 \mathrm{pc})$ in size $(\mathrm{FWHM})$, with the HCN emission coming from a somewhat smaller region. This im-
TABLE 4

INTEGRATED LINE INTENSITY RATIOS TOWARDS THE N113 PEAK

\begin{tabular}{lcccc} 
Ratio & SEST $^{\mathrm{a}}$ & ATCA 30m & ATCA 45m & ATCA 75m \\
\hline $\mathrm{HCO}^{+} / \mathrm{HCN}$ & $1.35 \pm 0.06$ & $1.27 \pm 0.09$ & $0.93 \pm 0.13$ & $0.90 \pm 0.29$ \\
$\mathrm{HCN} / \mathrm{HNC}$ & $2.81 \pm 0.21$ & $2.68 \pm 0.24$ & $3.49 \pm 0.71$ & $1.67 \pm 0.47$ \\
$\mathrm{C}_{2} \mathrm{H} / \mathrm{HCN}$ & $0.29 \pm 0.02$ & $0.30 \pm 0.05$ & $0.32 \pm 0.08$ & $<0.56$ \\
\hline \multicolumn{2}{c}{${ }^{\mathrm{a}}$ From Chin et al. (1997). }
\end{tabular}

plies a radius of $\sim 1.6 \mathrm{pc}$, comparable to that of the largest clumps which are found in Galactic massive star-forming regions (Mookeriea et al. 2004; Reid \& Wilson 2005), and much larger than the typical size $(\sim 0.1 \mathrm{pc})$ of a core (a condensation which forms only a single star or a multiple star system). We therefore believe it is appropriate to refer to this object as a "clump." We note, however, that it is common in the literature (e.g., Plume et al.1997) to use the term "massive cores" to refer to the birthplaces of the young stellar clusters where massive stars typically reside.

It is difficult to estimate the mass of the clump from the observed line fluxes given considerable uncertainties in the excitation, abundances, and optical depths of $\mathrm{HCO}^{+}$and $\mathrm{HCN}$. However, we can derive a virial mass as in $\$ 3.1 .2$ this time using $R=1.6 \mathrm{pc}$ and $\Delta v=5.5 \mathrm{~km} \mathrm{~s}^{-1}: M_{\mathrm{vir}}=151 R(\Delta v)^{2}=$ $7.3 \times 10^{3} M_{\odot}$, or about $10 \%$ of the total cloud mass inferred from the CO data. Alternatively, Gao \& Solomon (2004a) argue on the basis of large velocity gradient (LVG) calculations that the mass of dense gas traced by $\mathrm{HCN}$ satisfies

$$
M_{\text {dense }}\left(\mathrm{H}_{2}\right) \lesssim 25\left(\frac{L_{\mathrm{HCN}}}{\mathrm{K} \mathrm{km} \mathrm{s}^{-1} \mathrm{pc}^{-2}}\right) M_{\odot} .
$$

They obtain this result assuming an $\mathrm{HCN}$ abundance of $2 \times$ $10^{-8}$, a kinetic temperature of $20-50 \mathrm{~K}$ and an intrinsic $\mathrm{HCN}$ brightness temperature of $T_{b}>5 \mathrm{~K}$. Using this formula gives an upper limit of $1.3 \times 10^{4} M_{\odot}$ for the N113 clump, within a factor of 2 of the virial estimate. The agreement between the two mass estimates, along with the high densities implied by the detection of $\mathrm{HCN}, \mathrm{HCO}^{+}$, and $\mathrm{HNC}$, suggests that the assumption of virialization is reasonable. The average density of the clump is inferred to be $\left\langle n_{\mathrm{H}}\right\rangle \approx 10^{5} \mathrm{~cm}^{-3}$, close to the critical densities for $\mathrm{HCN}$ and $\mathrm{HCO}^{+}$.

\subsubsection{Line ratio analysis}

Figure 5 shows the integrated flux (in Jy $\mathrm{km} \mathrm{s}^{-1}$ ) for all four lines observed with ATCA as a function of the projected baseline. The equivalent curve for the continuum flux (in Jy, scaled down by 100) is also shown. Since the ATCA spacings are roughly in multiples of $15 \mathrm{~m}$, data have been averaged in bins of that width. As expected for a resolved source, the fluxes decrease on longer baselines, except for $\mathrm{C}_{2} \mathrm{H}$, which shows roughly equal fluxes on $30 \mathrm{~m}$ and $45 \mathrm{~m}$ baselines (it is not detected on longer baselines, however). The relatively faster decline seen for $\mathrm{HCO}^{+}$compared to $\mathrm{HCN}$ is responsible for the larger source size inferred from the visibility fitting (Table 3) and is corroborated by the Mopra maps. For the shorter ATCA baselines, we have tabulated several integrated line ratios, $I\left(\mathrm{HCO}^{+}\right) / I(\mathrm{HCN}), I(\mathrm{HCN}) / I(\mathrm{HNC})$, and $I\left(\mathrm{C}_{2} \mathrm{H}\right) / I(\mathrm{HCN})$, in Table 4 These are given alongside the SEST line ratios reported by Chin et al. (1997). Note that for $\mathrm{C}_{2} \mathrm{H}$ we only include the $87.3169 \mathrm{GHz}$ line in these calculations.

\section{DISCUSSION}




\subsection{Comparison with Galactic Clumps}

Recently a number of papers have analyzed the substructure of molecular clouds hosting massive star formation in our Galaxy (e.g., Shirlev et al. 2003; Faúndez et al. 2004). The clumps found in these studies have typical masses of 100-1000 $M_{\odot}$, sizes of $0.25-0.5 \mathrm{pc}$, and peak densities up to $10^{6} \mathrm{~cm}^{-3}$. The clump we have imaged in $\mathrm{N} 113$, with a radius $R \sim 1.6 \mathrm{pc}$ and mass $M \sim 10^{4} M_{\odot}$, lies at the upper end of this distribution in terms of size and mass. We believe our size estimate to be realistic: while the resolution of the ATCA maps $\left(\sim 4^{\prime \prime}\right)$ is only slightly smaller than the deconvolved size of the clump $\left(6^{\prime \prime}\right)$, the visibility amplitude as a function of baseline indicates that the source is well-resolved (Fig. 5). It is tempting to speculate that such massive clumps may be responsible for the formation of "populous" clusters of $10^{3}-10^{4}$ stars in the LMC (van den Bergh 1991).

Consistent with some previous studies of massive starforming regions (e.g. Plume et al. 1997), we do not find evidence of a size-linewidth relation of the form $\Delta v \propto R^{0.5}$ as seen in low-mass star-forming regions (Larson 1981). Indeed, the velocity dispersion of the clump is comparable to or even larger than that of the molecular cloud as a whole $\left(\sim 5 \mathrm{~km} \mathrm{~s}^{-1}\right)$. This may indicate a high level of turbulence, as found in massive star-forming cores in the Galaxy. However, we caution that the observed line width could be affected by optical depth effects, and confirmation of this result with an optically thin tracer is required.

\subsection{The Star-forming Enviroment in N113}

The overall picture that emerges of the interstellar medium near N113 is of an environment which has been strongly affected by recent star formation. In addition to the diffuse $\mathrm{H} \alpha$ seen in optical images, the linear sequence of 1-cm continuum sources, including two which are associated with $\mathrm{H}_{2} \mathrm{O}$ maser emission, point to the presence of young ionizing stars [Fig. 2 a)]. The brightest $\mathrm{H}_{2} \mathrm{O}$ maser is found near a relatively weak ( $\sim 4 \mathrm{mJy})$ continuum source (No. 4) southeast of the ATCA field, where there is still substantial $\mathrm{HCO}^{+}$and $\mathrm{CO}$ emission but no appreciable HCN. Moreover, referring to the 2MASS Point Source Catalog (Skrutskie et al. 2006), the associated near-infrared source is apparent in all three 2MASS bands $\left(m_{J}=13.87, m_{H}=13.46, m_{K}=12.71\right)$, suggesting it is relatively unobscured. These two lines of evidence point to an object that is somewhat more evolved than the brightest ( $\sim 66 \mathrm{mJy})$ of the continuum sources, which is near the peak of the molecular line emission and detected in $K$-band but not $J$-band by 2 MASS. This obscured source $\left(m_{J}>14.97\right.$, $\left.m_{H}=14.92, m_{K}=13.25\right)$ is shown by a solid square in Fig. 1 A third line of evidence is the presence of an $\mathrm{OH}$ maser in the former object, again suggesting a later stage of evolution.

Assuming the brightest continuum source emits optically thin free-free emission (spectral index -0.1 ), the measured fluxes at 24 and $86 \mathrm{GHz}$ (66 and $42 \mathrm{mJy}$ respectively) correspond to an ionizing photon output of $1.5-3 \times 10^{49} \mathrm{~s}^{-1}$ (Condon 1992), equivalent to one or two O6 stars (e.g., Turner et al. 1998). Although the measured fluxes suggest a spectral index of -0.3 instead of -0.1 , the larger calibration uncertainties of the $86 \mathrm{GHz}$ data (a result of higher $T_{\text {sys }}^{*}$ and relying on an $\mathrm{SiO}$ maser for gain calibration), possibly combined with resolving out some extended flux, could explain this discrepancy. The $86 \mathrm{GHz}$ image also appears to show an elongation to the north, whose significance is unclear but is interesting in light of the presence of an $\mathrm{H}_{2} \mathrm{O}$ maser $\sim 4^{\prime \prime}$ north of the $K$-band source. The maser and associated continuum emission could signify an outflow from the central ionizing source, but given their large projected distance from that source $(\sim 1 \mathrm{pc})$, are more likely to trace other ionizing stars in the same association.

Regardless of the exact nature of the radio continuum emission, its location only 3 .' $6^{\prime} \operatorname{arcsec}(0.9 \mathrm{pc})$ from the dense molecular clump will surely affect the lifetime of the clump. One possibility is that the pressure of the expanding $\mathrm{H}$ II region may compress the clump and thereby incite gravitational collapse. Whitworth et al. (1994) discuss how shocked gas layers generated by expanding $\mathrm{H}$ II regions tend to fragment into $\sim 7 M_{\odot}$ condensations which can become gravitationally unstable, yielding a self-propagating mode of star formation. Such a scenario might explain the alignment of starforming regions seen in the continuum. On the other hand, Gorti \& Hollenbach (2002) discuss how FUV radiation surrounding an $\mathrm{H}$ II region heats the clumpy molecular gas and eventually photoevaporates it, limiting the efficiency of star formation. A clump as massive as that inferred by our ATCA observations would not be easily photoevaporated, but might initially experience a shock compression due to heating and expansion of its outer layers. Observations of shock tracers in this region with Spitzer may be able to directly trace this process and assess its ability to trigger new star formation.

\subsection{Evidence for PDR Chemistry}

The large $\mathrm{HCO}^{+} / \mathrm{HCN}$ ratio observed with the shortest ATCA baseline (and in the Mopra data), decreasing on longer interferometer baselines, is consistent with the $\mathrm{HCO}^{+}$emission originating from a more extended region than HCN. Similar conclusions for the LMC were reached by Johansson et al. (1994), based on SEST mapping of the N159W cloud, and Heikkilä et al. (1999), based on small maps of several additional clouds. As pointed out by Heikkilä et al. (1999), two of the major chemical pathways for the formation of $\mathrm{HCO}^{+}$involve $\mathrm{C}^{+}$, which is expected to be abundant near the surfaces of clouds exposed to FUV radiation, i.e. in photon-dominated regions (PDRs). Thus, given the higher [C II]/CO flux ratios in the LMC (Mochizuki et al. 1994), and especially towards bright $\mathrm{H}$ II regions (Israel et al. 1996), $\mathrm{HCO}^{+}$emission likely traces lower density gas in the LMC in addition to wellshielded dense cores. The situation is less clear for $\mathrm{HCN}$ : while it can be produced by endothermic reactions between $\mathrm{CN}$ and $\mathrm{H}_{2}$ in the outer layers of a PDR, it is easily be dissociated back to form $\mathrm{CN}$ in the presence of strong FUV radiation (Sternberg \& Dalgarno 1995). We discussed above (\$3.2.3 the possibility that the more optically thin $\mathrm{HCN}$ arises from a "clump envelope" which is not fully imaged in our ATCA data. The larger size of the $\mathrm{HCO}^{+}$emission region then implies a somewhat larger envelope in this line than in $\mathrm{HCN}$.

$\mathrm{C}_{2} \mathrm{H}$ is also expected to be enhanced when $\mathrm{C}^{+}$is, and is especially strong relative to $\mathrm{CO}$ in the actively star-forming cloud N159W (Heikkilä et al. 1999). However, the SEST and ATCA observations show a roughly constant $I\left(\mathrm{C}_{2} \mathrm{H}\right) / I(\mathrm{HCN})$ from large to small scales, suggesting that $\mathrm{C}_{2} \mathrm{H}$ may trace dense gas rather than the PDR region. Such a result is rather surprising, given the relatively low dipole moment of $\mathrm{C}_{2} \mathrm{H}$, which should lead to its excitation in even low-density regions. An alternative explanation is that $\mathrm{C}_{2} \mathrm{H}$ appears less extended than $\mathrm{HCO}^{+}$because of lower optical depth. The hyperfine structure of $\mathrm{C}_{2} \mathrm{H}(N=1-0, J=3 / 2-1 / 2)$ consists of two lines $(F=2-1$ and $F=1-0)$ separated by $12 \mathrm{MHz}$. The integrated intensity ratio of the $2-1$ to $1-0$ line measured by 

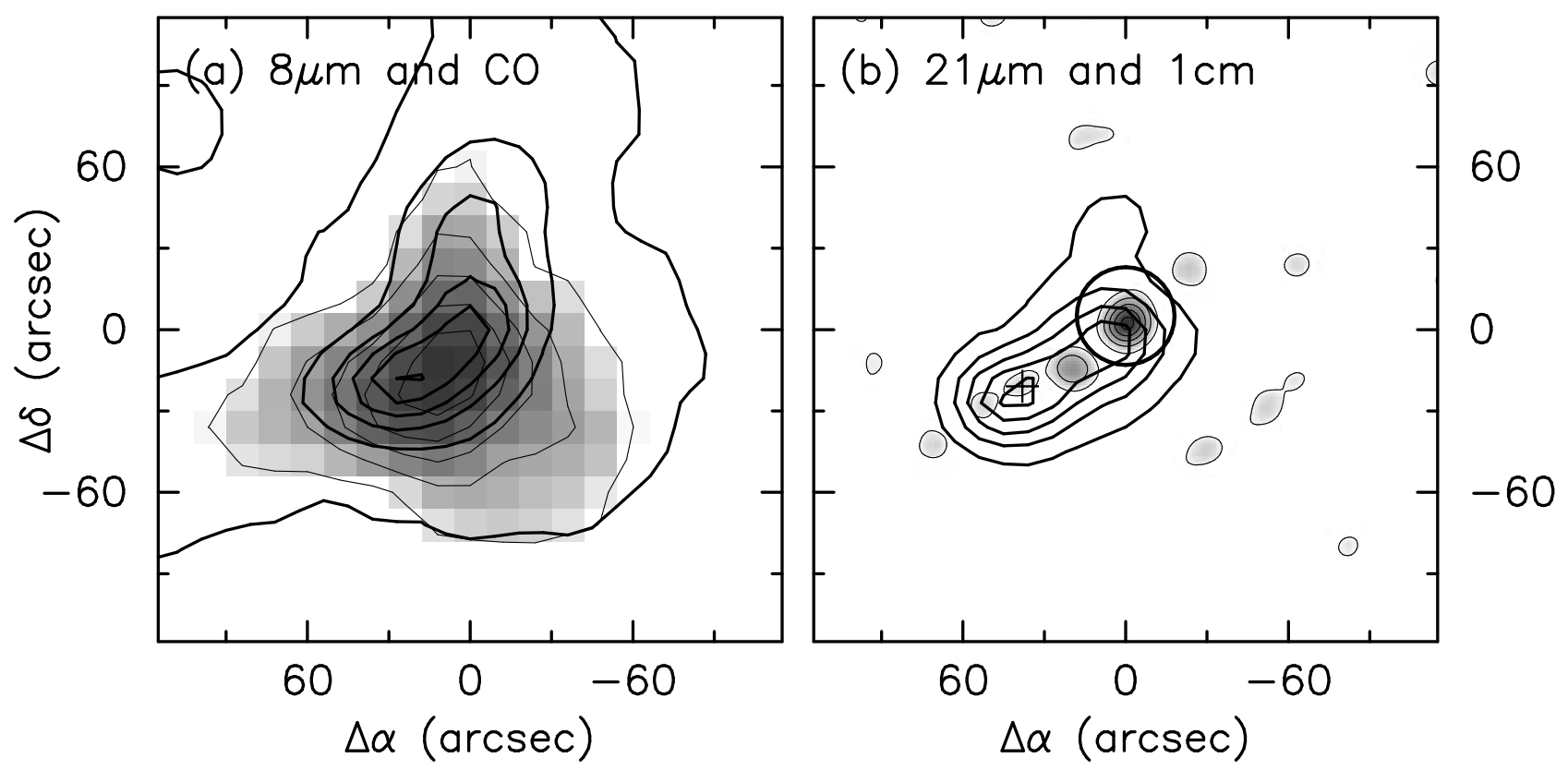

FIG. 6.- (a) $8 \mu \mathrm{m}$ MSX image (heavy contours) overlaid on the Mopra CO map from Fig.2(light contours and grayscale). Contour levels for the MSX image are spaced by $1.5 \times 10^{-10} \mathrm{~W} \mathrm{~cm}^{-2} \mathrm{sr}^{-1}$. (b) $21 \mu \mathrm{m}$ MSX image (heavy contours) overlaid on the $1 \mathrm{~cm}$ continuum map (light contours and grayscale). Contour levels for MSX image are spaced by $4 \times 10^{-10} \mathrm{~W} \mathrm{~cm}^{-2} \mathrm{sr}^{-1}$, while the continuum image has contours of $2 n^{2} \mathrm{mJy} \mathrm{bm}^{-1}$ ( $\left.n=1,2, \ldots\right)$. The ATCA pointing position is shown as a solid circle. The position of the bright $\mathrm{H}_{2} \mathrm{O}$ maser is shown by a cross.

SEST is 1.8 (Chin et al. 1997), close to the theoretical value of 2 (Tucker et al. 1974), and indicating that the opacity is relatively low. On the other hand, the $\mathrm{HCO}^{+} / \mathrm{H}^{13} \mathrm{CO}^{+}$intensity ratio towards N113 as measured by SEST is 34 (Chin et al. 1996), consistent with the isotopic ${ }^{12} \mathrm{C} /{ }^{13} \mathrm{C}$ ratio derived by Johansson et al. (1994) for N159. Thus, the optical depth in $\mathrm{HCO}^{+}$is likely to be small as well. More sensitive observations will be needed to draw firmer conclusions on how $\mathrm{C}_{2} \mathrm{H}$ is distributed relative to $\mathrm{HCN}$ and $\mathrm{HCO}^{+}$.

A more direct indicator of PDR conditions may be the infrared emission at $8 \mu \mathrm{m}$, which is primarily attributed to large organic molecules known as polycyclic aromatic hydrocarbons (PAHs) heated by far UV radiation in the outer layers of the cloud. As Figure 6 a) indicates, the distribution of $6.8 \mu \mathrm{m}-$ $10.8 \mu \mathrm{m}$ emission near N113, as measured by the MSX satellite (Price et al. 1998), closely matches the CO distribution in extent. This provides further evidence that the extended $\mathrm{HCO}^{+}$emission, which follows the $\mathrm{CO}$ distribution, is enhanced due to PDR chemistry. The $21 \mu \mathrm{m}$ emission, which traces heated dust, is peaked slightly to the southeast of the $\mathrm{PAH}$ emission, and corresponds well to the $1.3-\mathrm{cm}$ radio continuum emission [Figure 6(b)].

\subsection{Comparison of $H C N$ and $H N C$}

Aside from a highly uncertain measurement for the $45 \mathrm{~m}$ baseline, the HCN/HNC intensity ratio appears to decrease on longer baselines, which sample smaller scale structure. This would a confirm a prediction by Chin et al. (1997), that the ratio would be large in warm gas subject to strong UV heating but approach unity in cloud cores. The difference may be due to additional neutral-neutral pathways for $\mathrm{HCN}$ formation that occur in a partially ionized medium, such as $\mathrm{CH}_{2}+\mathrm{N} \rightarrow$ $\mathrm{HCN}+\mathrm{H}$ (Goldsmith et al. 1981). These produce $\mathrm{HCN}$ faster than the equivalent reactions $\left(\mathrm{NH}_{n}+\mathrm{C} \rightarrow \mathrm{HNC}+\mathrm{H}_{n-1}\right)$ produce $\mathrm{HNC}$, due to the higher proportion of $\mathrm{CH}_{n}$ radicals and the tendency for $\mathrm{C}$ to be ionized in PDRs. However, the abil- ity of such reactions to produce $\mathrm{HCN} / \mathrm{HNC}$ abundance ratios much different from unity has been questioned (Herbst et al. 2000). In any case, the observed ratios are consistent with the HCN/HNC ratios of $\sim 1-6$ observed in starburst galaxies (Aalto et al. 2002). A major uncertainty in interpreting intensity ratios as abundance ratios is the assumption of low optical depth. The decreasing HCN/HNC on small scales coincides with an apparent increase in the HCN opacity, which could affect the line profiles through saturation or self-absorption.

\section{SUMMARY \& CONCLUSIONS}

We have presented high-resolution imaging of $\mathrm{HCO}^{+}$, $\mathrm{HCN}, \mathrm{HNC}$, and $\mathrm{C}_{2} \mathrm{H}$ towards the $\mathrm{N} 113 \mathrm{H}$ II region in the LMC, performed with the ATCA interferometer, as well as single-dish mapping of the $\mathrm{CO}, \mathrm{HCO}^{+}$, and $\mathrm{HCN}$ lines with Mopra. Our main conclusions are summarized below.

1. On scales of several arcminutes, the molecular cloud $\left(M \sim 10^{5} M_{\odot}\right)$ shows a weak velocity gradient which is shared with an H I filament which encompasses it. Several radio continuum sources lie along the center of the filament, making it the locus of recent star formation.

2. Near the brightest continuum source, which we interpret as a compact $\mathrm{H}$ II region, we detect a massive clump of dense molecular gas whose peak is slightly offset from the $3 \mathrm{~mm}$ continuum peak. The clump has a radius of $\sim 1 \mathrm{pc}$, and if in virial equilibrium, a mass of $\sim 10^{4} M_{\odot}$, about $10 \%$ of the cloud's mass. This would imply a density of $\sim 10^{5} \mathrm{~cm}^{-3}$. With the ionizing flux of 1-2 O6 stars, the compact $\mathrm{H}$ II region may possibly trigger the formation of new stars in the clump.

3. While the clump-when combined with a more diffuse envelope inferred from visibility fitting - may account for most of the $\mathrm{HCO}^{+}$and $\mathrm{HCN}$ emission within the ATCA primary beam, the existence of additional 
clumps within the cloud is not excluded given the small extent of the region mapped thus far with the ATCA.

4. A decreasing $\mathrm{HCO}^{+} / \mathrm{HCN}$ ratio on longer interferometer baselines, which probe smaller scales, suggests that the $\mathrm{HCO}^{+}$emanates in part from a more diffuse region then the HCN. This is consistent with the larger spatial extent of the $\mathrm{HCO}^{+}$emission in the Mopra map and its agreement with the $\mathrm{CO}$ and $8 \mu \mathrm{m}$ morphology.

5. We also see evidence for a decrease in the HCN/HNC ratio on smaller scales. This may reflect a lower ratio in cold, dense regions, as found in the Galaxy, but the interpretation may be clouded by an increased opacity in the HCN line.

These observations demonstrate that maps of LMC molecular clouds on scales of a few pc can be used to investigate the internal structure and chemistry of clouds for comparison with Galactic samples. With the ATCA now covering a frequency range of $85-105 \mathrm{GHz}$ with five antennas, further observations will soon achieve better sensitivity for a larger number of molecular lines, allowing for a more detailed comparison with PDR models. Ultimately, the ability to observe both low and high excitation lines at high resolution with the Atacama Large Millimeter Array (ALMA) will facilitate a comprehensive understanding of the properties of molecular clouds in an external galaxy.

We thank L. Staveley-Smith and S. Kim for providing the $\mathrm{H}$ I datacube used for comparison, and M. Burton and C. Henkel for providing helpful comments on the manuscript. Paul Ho and an anonymous referee also suggested improvements for the revised version. We thank R. Sault and the staff of the Narrabri observatory for assistance and troubleshooting at both ATCA and Mopra. The Mopra data were obtained in the UNSW time allocation with the kind permission of $\mathrm{M}$. Burton. TW acknowledges support from an ARC-CSIRO Linkage Postdoctoral Fellowship. YNC acknowledges financial support through National Science Council of Taiwan grant NSC 89-2119-M-032-001. This publication makes use of data products from the Two Micron All Sky Survey, which is a joint project of the University of Massachusetts and the Infrared Processing and Analysis Center/California Institute of Technology, funded by the National Aeronautics and Space Administration and the National Science Foundation. This research has made use of NASA's Astrophysics Data System Bibliographic Services. The Digitized Sky Surveys were produced at the Space Telescope Science Institute under U.S. Government grant NAG W-2166.

\section{REFERENCES}

Aalto, S., Polatidis, A. G., Hüttemeister, S., \& Curran, S. J. 2002, A\&A, 381, 783

Bica, E., Claria, J. J., \& Dottori, H. 1992, AJ, 103, 1859

Blitz, L. 1991, in The Physics of Star Formation and Early Stellar Evolution, ed. C. J. Lada \& N. D. Kylafis (Dordrecht: Kluwer), 3

Brooks, K. J. \& Whiteoak, J. B. 1997, MNRAS, 291, 395

Chin, Y.-N., Henkel, C., Millar, T. J., Whiteoak, J. B., \& Mauersberger, R. 1996, A\&A, 312, L33

Chin, Y.-N., Henkel, C., Whiteoak, J. B., Millar, T. J., Hunt, M. R., \& Lemme, C. 1997, A\&A, 317, 548

Churchwell, E., Nash, A. G., \& Walmsley, C. M. 1984, ApJ, 287, 681

Condon, J. J. 1992, ARA\&A, 30, 575

Dame, T. M., Hartmann, D., \& Thaddeus, P. 2001, ApJ, 547, 792

Faúndez, S., Bronfman, L., Garay, G., Chini, R., Nyman, L.-Å., \& May, J. 2004, A\&A, 426, 97

Gao, Y. \& Solomon, P. M. 2004a, ApJS, 152, 63

-. 2004b, ApJ, 606, 271

Goldsmith, P. F., Langer, W. D., Ellder, J., Kollberg, E., \& Irvine, W. 1981, ApJ, 249, 524

Gorti, U. \& Hollenbach, D. 2002, ApJ, 573, 215

Graedel, T. E., Langer, W. D., \& Frerking, M. A. 1982, ApJS, 48, 321

Harju, J. 1989, A\&A, 219, 293

Heikkilä, A., Johansson, L. E. B., \& Olofsson, H. 1999, A\&A, 344, 817

Henize, K. G. 1956, ApJS, 2, 315

Herbst, E., Terzieva, R., \& Talbi, D. 2000, MNRAS, 311, 869

Hirota, T., Yamamoto, S., Mikami, H., \& Ohishi, M. 1998, ApJ, 503, 717

Huettemeister, S., Henkel, C., Mauersberger, R., Brouillet, N., Wiklind, T., \& Millar, T. J. 1995, A\&A, 295, 571

Israel, F. P., Maloney, P. R., Geis, N., Herrmann, F., Madden, S. C., Poglitsch, A., \& Stacey, G. J. 1996, ApJ, 465, 738

Johansson, L. E. B., Olofsson, H., Hjalmarson, A., Gredel, R., \& Black, J. H. 1994, A\&A, 291, 89

Kim, S., Staveley-Smith, L., Dopita, M. A., Sault, R. J., Freeman, K. C., Lee, Y., \& Chu, Y.-H. 2003, ApJS, 148, 473

Ladd, N., Purcell, C., Wong, T., \& Robertson, S. 2005, PASA, 22, 62

Larson, R. B. 1981, MNRAS, 194, 809
Lazendic, J. S., Whiteoak, J. B., Klamer, I., Harbison, P. D., \& Kuiper, T. B. H. 2002, MNRAS, 331, 969

Mochizuki, K., Nakagawa, T., Doi, Y., Yui, Y. Y., Okuda, H., Shibai, H., Yui, M., Nishimura, T., \& Low, F. J. 1994, ApJ, 430, L37

Mookerjea, B., Kramer, C., Nielbock, M., \& Nyman, L.-Å. 2004, A\&A, 426, 119

Panagia, N. 1999, in IAU Symp. 190: New Views of the Magellanic Clouds, 549

Plume, R., Jaffe, D. T., Evans, N. J., Martin-Pintado, J., \& Gomez-Gonzalez, J. 1997, ApJ, 476, 730

Price, S. D., Tedesco, E. F., Cohen, M., Walker, R. G., Henry, R. C., Moshir, M., Paxton, L. J., \& Witterborn, F. C. 1998, in IAU Symp. 179: New Horizons from Multi-Wavelength Sky Surveys, ed. B. J. McLean, D. A. Golombek, J. J. E. Hayes, \& H. E. Payne, 115

Reid, M. A. \& Wilson, C. D. 2005, ApJ, 625, 891

Rosolowsky, E., Engargiola, G., Plambeck, R., \& Blitz, L. 2003, ApJ, 599, 258

Rubio, M., Lequeux, J., \& Boulanger, F. 1993, A\&A, 271, 9

Sault, R. J., Teuben, P. J., \& Wright, M. C. H. 1995, in Astronomical Data Analysis Software and Systems IV, ASP Conference Series Vol. 77, ed. R. A. Shaw, H. E. Payne, \& J. J. E. Hayes (San Francisco: ASP), 433

Schilke, P., Walmsley, C. M., Pineau Des Forets, G., Roueff, E., Flower, D. R., \& Guilloteau, S. 1992, A\&A, 256, 595

Shirley, Y. L., Evans, N. J., Young, K. E., Knez, C., \& Jaffe, D. T. 2003, ApJS, 149,375

Skrutskie, M. F. et al. 2006, AJ, 131, 1163

Sternberg, A. \& Dalgarno, A. 1995, ApJS, 99, 565

Strong, A. W. \& Mattox, J. R. 1996, A\&A, 308, L21

Tucker, K. D., Kutner, M. L., \& Thaddeus, P. 1974, ApJ, 193, L115

Turner, J. L., Ho, P. T. P., \& Beck, S. C. 1998, AJ, 116, 1212

van den Bergh, S. 1991, ApJ, 369, 1

Whiteoak, J. B. \& Gardner, F. F. 1986, MNRAS, 222, 513

Whitworth, A. P., Bhattal, A. S., Chapman, S. J., Disney, M. J., \& Turner, J. A. 1994, MNRAS, 268, 291 